\title{
Optical Properties of Carbon Nanotubes under External Electric Fields
}

\author{
M. Pacheco, Z. Barticevic*, A. Latgé ${ }^{\dagger}$, and C. G. Rocha ${ }^{\dagger}$ \\ *Departamento de Física, Universidad Tecnica F. Santa Maria, \\ Av. Frederico Santa Maria, 6090, Viña del Mar, Chile and \\ ${ }^{\dagger}$ Instituto de Física, Universidade Federal Fluminense, Av. Litorânea sn, 24210-340, Niterói, RJ, Brazil
}

Received on 4 April, 2005

\begin{abstract}
We study the optical properties of carbon nanotubes within the single-band tight-binding approximation. The great number of one-dimensional-like subbands of the nanotubes lead to typical van Hove singularities on the local density of states. When a tube is under the influence of a laser beam, optical transitions are allowed between Van Hove singularities and they can be observed experimentally in absorption spectra. External magnetic and electric fields modify the energy spectrum of carbon nanotubes inducing changes on the optical phenomena at low frequencies. Results for the inter-band absorption coefficient are shown for nanotubes under an additional external electrostatic potential for parallel light polarization. This is an important problem to understand since low energy laser beams are being used to identify the chirality and diameter of the tubes and their electronic character.
\end{abstract}

Keywords: Optical properties; Carbon nanotubes; Single-band tight-binding approximation

\section{INTRODUCTION}

Although a large number of application of carbon nanotubes (CNs) in the industry and in optoelectronic devices are being proposed nowadays their implementations require mechanisms able to synthesize even more pristine CNs samples. Also, the correct characterization $[1,2]$ of tubes in general continues being a challenge task to overcome. Raman scattering methodologies [3, 4] are one of the most promising experimental approach that allow the characterization of the physical properties of CNs. The Raman spectroscopy is the inelastic scattering of the light by the sample in which a transfer of energy and momentum between the laser beam and the medium (and vice versa) occurs[5, 6]. The analysis of Raman spectra from $\mathrm{CN}$ bundles, allow ones to determine the diameter distribution and to infer the degree of alignment of the light polarization with the nanotubeś axis[7, 8]. We may also extracted $\mathrm{CNs}$ projected images of $\mathrm{CNs}$ [9]. Besides spectroscopy measurements, many theoretical works are directed to understand the main optical activities in CNs such as the dipole selection rules of optical transitions, depolarization effects and photoluminescence[10-13] phenomena predicted in the resonance Raman results via optical absorption formula considering low laser energies.

Optical absorption spectra as a function of the laser beam energy with polarization guided parallel to the nanotube axis may be calculated considering transitions from a $2 p$ orbital of one atom to its neighboring ( $\pi-\pi^{*}$ transitions) within the tight binding formalism[10, 11]. The time dependent perturbation theory provide the optical matrix elements for a dipole absorption between an initial valence and a final conduction state. It was shown that the parallel polarized light induces electronic transitions from $E_{\mu}^{v} \rightarrow E_{\mu^{\prime}}^{c}$ for energy sub-bands with $\mu=\mu^{\prime}$, i. e., the allowed transitions occur between Van Hove Singularities localized symmetrically around the Fermi energy $[10,14]$.

In this work, we calculate the absorption spectra of straight carbon nanotubes under the presence of an applied transversal electrical field and analyze the changes on the corresponding electronic properties. The action of external electric field alters the electronic character of the nanostructures leading in some cases to metal-insulator transitions[15-17]. These effects are also expected to be reflected in the absorption spectra. The understanding of such changes and its manipulation are quite important for using CNs in electronic devices.

\section{THEORY AND RESULTS}

The electronic structure of $\mathrm{CNs}$ is calculated following a single $\pi$-band tight binding approach, considering only nearest-neighbor interactions. The energy spectrum of a graphite sheet in this approximation can be obtained by expanding the wave function as a linear combination of atomic orbitals

$$
\begin{array}{r}
\psi(\vec{r})=\sum_{n_{1}, n_{2}}\left[C_{n_{1}, n_{2}}^{A} \phi_{A}^{S}\left(\vec{r}-\vec{R}_{n_{1}, n_{2}}\right)+\right. \\
\left.C_{n_{1}, n_{2}}^{B} \phi_{B}^{S}\left(\vec{r}-\vec{R}_{n_{1}, n_{2}}-\vec{\chi}_{B}\right)\right]
\end{array}
$$

where $\vec{R}_{n_{1} n_{2}}=n_{1} \vec{a}_{1}+n_{2} \vec{a}_{2}$ denotes the position of a carbon atom $A$ and $\vec{\chi}=-\left(\vec{a}_{1}+\vec{a}_{2}\right) / 3$ the position of the corresponding atom $\mathrm{B}$ in the same cell. The hexagonal lattice unit vectors are given by $\vec{a}_{1}=a_{c c} \frac{\sqrt{3}}{2}(1, \sqrt{3})$ and $\vec{a}_{2}=a_{c c} \frac{\sqrt{3}}{2}(-1, \sqrt{3})$, with $a_{c c}=1.42 \AA$.

The unit cell of straights $\mathrm{CNs}$ are specified by a chiral vector $\vec{C}_{h}=n \vec{a}_{1}+m \vec{a}_{2}$ in the circumferential direction and a translational vector $\vec{T}=p \vec{a}_{1}+q \vec{a}_{2}$, along the longitudinal direction. For zigzag CNs the translational symmetry along the y-direction is preserved and the coefficients $C^{A}$ and $C^{B}$ may be redefined in terms of a wave vector $k_{y}$. One then gets the following set of coupled equations 


$$
\begin{gathered}
\left(\varepsilon-\varepsilon_{A}(i)\right) A_{i}-\gamma\left[B_{i}+e^{\frac{i 3}{2} k_{y} a_{c c}}\left(B_{i+1}+B_{i-1}\right)\right]=0 \\
\left(\varepsilon-\varepsilon_{B}(i)\right) B_{i}-\gamma\left[A_{i}+e^{\frac{-i}{2} k_{y} a_{c c}}\left(A_{i+1}+A_{i-1}\right)\right]=0 .
\end{gathered}
$$

with $\mathrm{i}=1, \ldots, 2 \mathrm{n}$. The wave vectors $k_{y}$ are defined within the interval $-\frac{\pi}{T_{y}}<k_{y}<\frac{\pi}{T_{y}}$, and the magnitude of the translation vector along the tube is given by $T=3 a_{c c}$. The effect of the electric field is included into the on-site energies of the carbon atoms which are given by

$$
\varepsilon_{A(B)}(i)=-V(n) \cos \left(\theta_{i}^{A(B)}\right),
$$

where the electrostatic potential energy along the tube crosssection $V(n)$ and the electric field value are related by $V(n)=$ $e F n \sqrt{3} a_{c c} / \pi$. The azimuthal angle $\theta_{i}$ is defined by the electric field direction and the position of the carbon atoms along the cylindrical surface of the tube and is given by $\theta_{i}^{A(B)}=(i-$ 1) $\pi / n$.

The optical absorption spectra is given within the dipolar approximation by

$$
A(\omega)=A_{o}\left|\hat{\varepsilon} \cdot\left\langle\psi_{f}^{c}|\nabla| \psi_{i}^{\nu}\right\rangle\right|^{2} \delta\left(E_{i}-E_{f}-\hbar \omega\right)
$$

with $\hat{\varepsilon}$ being the photon polarization (considered parallel to the axis tube) and $\psi_{f, i}^{c, v}$ is the final (initial) electronic wave function on the conduction (valence) band [10,11, 13]. The knowledge of the $A$ and $B$ coefficients allow us to construct the electronic wave functions and, consequently, resolve the dipole vector expression. One should notice that the delta function is changed by a Lorentzian distribution written in terms of $\omega$, the incident laser energy.
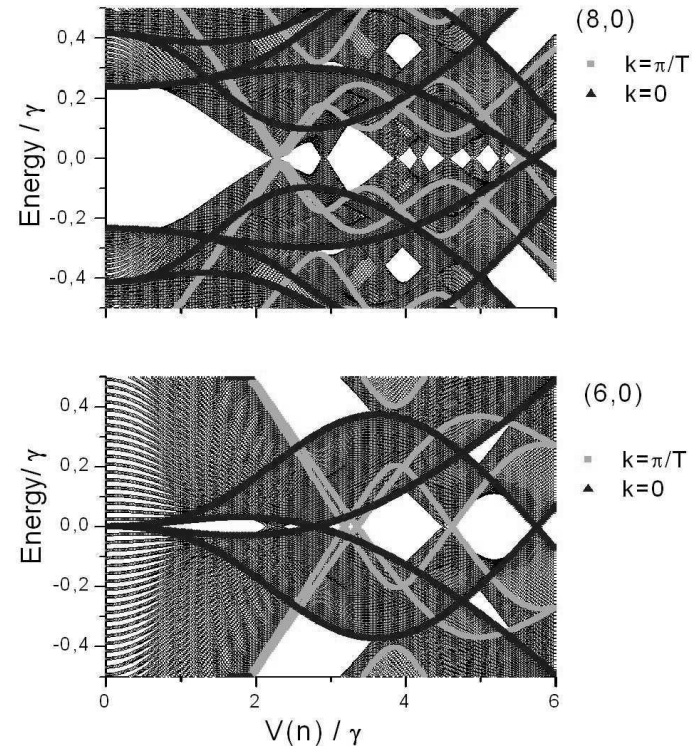

FIG. 1. Energy spectrum as a function of the electric field energy for a $(8,0)$ semiconductor and $(6,0) \mathrm{CNs}$ with $120 \mathrm{k}_{y}$-numbers.

With the purpose of illustrating the main effects of an electric field on a zigzag nanotube we display in Fig. 1 the energy spectra for a semiconductor $(8,0)$ and metallic $(6,0)$ CNs as a function of the field energy. One expects that the same kind of information may be obtained from the determination of the optical absorption spectrum. Some particular allowed values of the $k$ vector, along the circumferential direction, are marked by the colored symbols (triangled and squared lines). In what follows all the energies are written in units of hopping parameter $(\gamma \approx-2.66 \mathrm{eV})$. For low field values, the energy levels laying closer to the Fermi energy are almost unaffected whereas the states more distant in energy, mainly those degenerated at zero field, are strongly affected resulting in splitting and crossing of levels for increasing electric field intensities.

Alternatively, by turning on a laser beam polarized parallel to the tube axis one may extract those effects from the optical absorption spectrum. Results for the absorption coefficients [equation 4] of a $(8,0) \mathrm{CN}$ are shown in Fig. 2(a) considering low intensity field energies. Each one of the curves corresponds to an electric field energy value varying from $\frac{V}{\gamma}=0$ to 0.6 , spaced by 0.02 . The peaks correspond to the allowed optical transitions that may occur between maximum or minimum points of the $\mathrm{CN}$ sub-bands. As foreseen by the corresponding $(8,0)$ energy spectrum, the low-energy absorption peaks keep unchanged on energy while robust modifications occur for more excited levels. Even for these low intensity ranges, the electric field reduces the symmetry operations allowed for the tube unit cell in the absence of external fields, leading to degeneracy broken of some excited states. The lifting of degeneracies turns possible new optical transitions due to new singularity peaks around $\gamma$ and $-\gamma$ exhibited in the local density of states (LDOS) shown in figure 2(b).
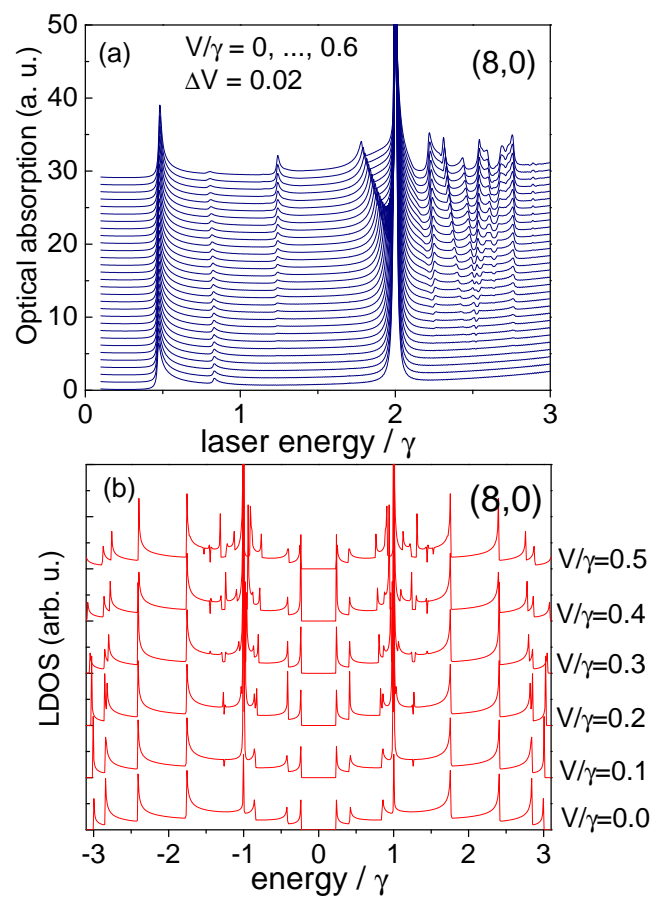

FIG. 2. (a) Optical absorption spectrum as a function of the laser energy for a $(8,0)$ semiconductor $\mathrm{CN}$ with electric field energy values $V / \gamma=0$ to 0.6 (steps of 0.02 ). (b) LDOS of $(8,0)$ as a function of the energy for different values of electric field energy. 


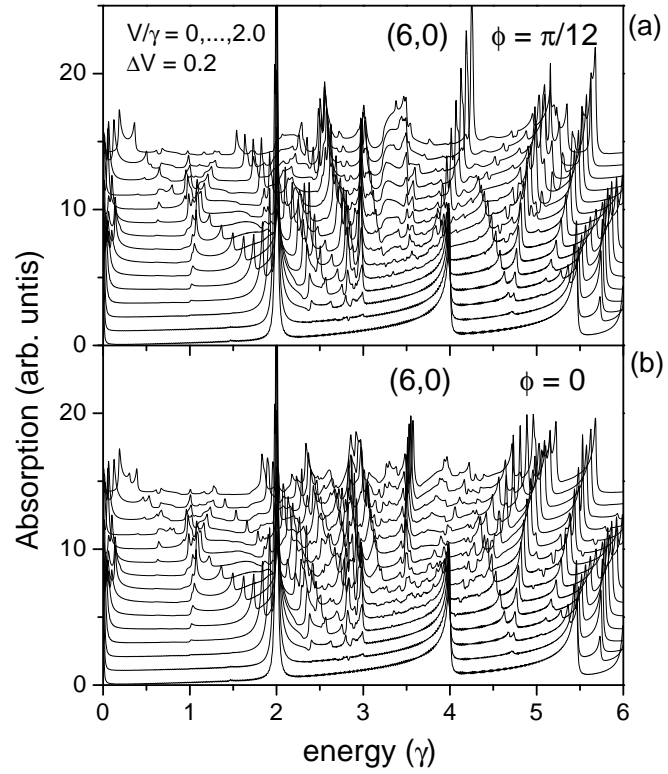

FIG. 3. Optical absorption coefficients as a function of the laser energy for a $(6,0)$ metallic $\mathrm{CN}$ with electric field energy varying from $V / \gamma=0$ to 2.0 (steps of 0.2 ), for a dephasing angle of (a) $\phi=0$ and (b) $\phi=\pi / 12$.

We may also induce changes on the optical transitions simulating an electric field rotation between two equivalent inplane atomic positions laying at one single ring of the tube. This is described by adding an extra phase, $\phi$, in the azimuthal angle in the site energy expression. One verifies that some electronic states of CNs depend on this dephasing angle[15]. Fig. 3 shows the optical absorption spectra for a $(6,0)$ metallic $\mathrm{CN}$ varying the electric field energy from 0 to 2.0 (steps equal to 0.2 ). Comparing the spectra corresponding to $\phi=\pi / 12$ and $\phi=0$, we may observe that some absorption transitions are shifted in energies and even smoothed whereas others are en- hanced when the rotation is performed. In the example shown it is clear the modification suffered by the absorption coefficient for photon energies next to $4 / \gamma$. Therefore, a simple rotation performed on the external electric field direction may be used as an extra tool to manipulate the electronic levels, and consequently, the optical properties. Of course, one is aware that for a quantitative description of the optical absorption spectra it is necessary to go beyond the single one-band tight binding approach used here. Work in that direction will be published elsewhere.

Summing up we have addressed a theoretical qualitative study on the optical properties of carbon nanotubes considering a simple tight-binding scheme. Inter-band absorption coefficient have been calculated for the tubes under an additional external electrostatic potential considering a parallel light polarization. At low frequency it was shown that the external electric field modify the energy spectrum of carbon nanotubes inducing changes on the optical phenomena. Further absorption coefficient modifications are also obtained by considering an extra phase corresponding to the relative direction of the applied fields and the in-plane atomic distribution.

One may use these studies to intentionally monitor appropriate configurations of photon absorption and also to characterize tubes in relation to their chirality and diameters, such as the actual experimental realizations.

\section{Acknowledgments}

This work was supported by the Brazilian Agencies CNPq, CAPES, FAPERJ, Instituto do Milênio, and PRONEX-CNPqFAPERJ grant $171.168-200$, and also by the Iniciativa Cientifica Milenio P02-054-F, Fondecyt 1050521 and 7050240.
[1] R. Saito, A. Gruneis, G. G. Samsonidze, V. W. Brar, G. Dresselhaus, M. S. Dresselhaus, A. Jorio, L. G. Cancado, C. Fantini, M. A. Pimenta, and A. G. Souza, New Journal of Physics 5, 157 (2003).

[2] H. Kataura, Y. Kumazawa, Y. Maniwa, I. Uemezu, S. Suzuki, Y. Ohtsuka, and Y. Achiba, Synth. Met. 103, 2555 (1999).

[3] M. S. Dresselhaus, G. Dresselhaus, A. Jorio, A. G. Souza Filho, M. A. Pimenta, and R. Saito, Acc. Chem. Res. 35, 1070 (2002).

[4] A. G. Souza, A. Jorio, J. H. Hafner, C. M. Lieber, R. Saito, M. A. Pimenta, G. Dresselhaus, and M. S. Dresselhaus, Phys. Rev. B 63, 241404 (2001).

[5] A. Jorio, M. A. Pimenta, A. G. Souza, R. Saito, G. Dresselhaus, and M. S. Dresselhaus, New Journal of Physics 5, 139 (2003).

[6] V. G. Hadjiev, M. N. Iliev, S. Arepalli, P. Nikolaev, and B. S. Files, Appl. Phys. Lett. 78, 3193 (2001).

[7] H. M. Cheng, F. Li, X. Sun, S. D. M. Brown, M. A. Pimenta, A. Marucci, G. Dresselhaus, and M. S. Dresselhaus, Chem. Phys. Lett. 289, 602 (1998).

[8] G. S. Duesberg, I. Loa, M. Burghard, K. Syassen, and S. Roth, Phys. Rev. Lett. 85, 5436 (2000).
[9] A. Hartschuh, E. J. Sanchez, X. S. Xie, L. Novotny, Phys. Rev. Lett. 90, 095503 (2003).

[10] A. Gruneis, R. Saito, Ge. G. Samsonidze, T. Kimura, M. A. Pimenta, A. Jorio, A. G. Souza Filho, G. Dresselhaus, and M. S. Dresselhaus, Phys. Rev. B 67, 165402 (2003).

[11] Ge. G. Samsonidze, A. Gruneis, R. Saito, A. Jorio, A. G. Souza Filho, G. Dresselhaus, and M. S. Dresselhaus, Phys. Rev. B 69, 205402 (2003).

[12] I. Bozović, N. Bozović, and M. Damnjanović, Phys. Rev. B 62, 6971 (2000).

[13] M. F. Lin, Phys. Rev. B 62, 13153 (2000).

[14] J. Jiang, R. Saito, A. Grüneis, G. Dresselhaus, and M. S. Dresselhaus, Carbon 42, 3169 (2004).

[15] C. G. Rocha, M. Pacheco, Z. Bartcevic, and A. Latgé, Phys. Rev. B 70, 233402 (2004).

[16] X. Zhou, H. Chen, and O. Y. Zhong-can, J. Phys. Condens. Matt. 13, L635 (2001).

[17] Y. H. Kim and K. J. Chang, Phys. Rev. B 64, 153404 (2001). 